\title{
Atomic inner-shell radiation seeded free-electron lasers
}

\author{
Han Zhang, ${ }^{1,2}$ Kai Li, ${ }^{2,3}$ Jiawei Yan, ${ }^{2,3}$ Haixiao Deng, ${ }^{2, *}$ and Baoyuan Sun ${ }^{1, \dagger}$ \\ ${ }^{1}$ School of Nuclear Science and Technology, Lanzhou University, Lanzhou 730000, China \\ ${ }^{2}$ Shanghai Institute of Applied Physics, Chinese Academy of Sciences, Shanghai 201800, China \\ ${ }^{3}$ University of Chinese Academy of Sciences, Beijing 100049, China
}

(Received 21 March 2018; published 3 July 2018)

\begin{abstract}
In order to effectively improve the output quality of x-ray free electron laser (XFEL), we theoretically propose an XFEL scheme seeded by atomic inner-shell laser. Atomic inner-shell lasers based on neutral atoms and pumped by XFEL have been experimentally demonstrated [Rohringer et al., Nature (London) 481, 488 (2012), Yoneda et al., Nature (London) 524, 446 (2015)], which produced sub-femtosecond X-ray pulses with increased temporal coherence. It shows that, by using the inner-shell laser as a seed to modulate the electron bunch, very stable and almost fully-coherent short-wavelength XFEL pulses can be generated. The proposed scheme holds promising prospects in x-ray wavelengths, and even shorter.
\end{abstract}

DOI: 10.1103/PhysRevAccelBeams.21.070701

\section{INTRODUCTION}

The generation of lasers has brought great benefits to physics, chemistry, and biology researches. These researchers have aimed to produce short-wavelength lasers that generate coherent $\mathrm{x}$-rays $[1,2]$. The shorter the wavelength, the better the imaging resolution of the laser and the shorter the pulse duration, leading to better temporal resolution in probe measurements. Recently, x-ray free electron lasers (XFEL) based on self-amplified spontaneous emission (SASE) $[3,4]$ have made it possible to generate a hard $\mathrm{x}$-ray laser (that is, photon energy is of the order of ten kiloelectronvolts) in an ångström-wavelength regime $[5,6]$. Nevertheless, this process starts from the electron beam shot noise and creates light pulses of limited temporal coherence [7]. As is well known, both narrow bandwidth and excellent longitudinal coherence are very important for ultrafast x-ray spectroscopy [8], x-ray quantum optics [9], and fundamental physics applications. In order to obtain fully coherent x-ray light pulses, a variety of new lasing schemes have been proposed and already demonstrated. For example, direct-seeding [10], self-seeding [11-14], high-gain harmonic generation [15-20], echo-enhanced harmonic generation [21], phase-merging enhanced harmonic generation [22,23], x-ray free-electron laser oscillator [24-26], in particular the self-seeding method is the most widely used scheme in hard XFEL facilities.

\footnotetext{
*denghaixiao@sinap.ac.cn

sunby@1zu.edu.cn
}

Published by the American Physical Society under the terms of the Creative Commons Attribution 4.0 International license. Further distribution of this work must maintain attribution to the author(s) and the published article's title, journal citation, and DOI.
However, the large pulse-to-pulse energy jitter and limited spectral brightness enhancement prevent it from further developments.

An alternative approach to create a pulse source in the $\mathrm{X}$-ray regime is to use a laser to pump an atomic inner-shell $\mathrm{X}$-ray laser (XRL). The first inner-shell XRL in the kiloelectronvolt regime was proposed in 1967 and is based on establishing a population inversion by rapid photoionization of an inner-shell electron [27]. The photoionization scheme was extensively studied in theory for different gain materials and pump sources in the x-ray regime [28-31]. However, the lack of sufficiently fast and intense $x$-ray sources has so far precluded the realization of the photoionization scheme in the $\mathrm{x}$-ray regime. The introduction of XFEL makes it possible to pump new atomic innershell XRL [32-35] with ultrashort pulse duration, extreme spectral brightness, and full temporal coherence. Experimentally, in 2012, An atomic laser based on neon atoms and pumped by a soft XFEL has been achieved at a wavelength of 14.6 ångströms in Linac Coherent Light Source [36]; in 2015, the SPring-8 Angstrom Compact Free Electron Laser use a copper target and report a hard X-ray atomic inner-shell laser operating at a wavelength of 1.5 ångströms [37]. Experimental results show that temporal coherence was greatly improved in the pumped neon $(\mathrm{Ne})$ and copper $(\mathrm{Cu})$ medium using XFEL.

In this work, we propose a scheme that combines selfseeding method and atomic inner-shell XRL. First, we use SASE XFEL as a pump source to pump a given neutral atom, which can produce a longitudinal coherent inner-shell XRL. Then this inner-shell XRL is used as a seed laser to modulate the electron beam, and generate a fully-coherent, shortwavelength and stable XFEL. The principle of our scheme, including the seeded XFEL and x-ray lasing in core excited atom are described in Sec. II. The small-signal gain and the 


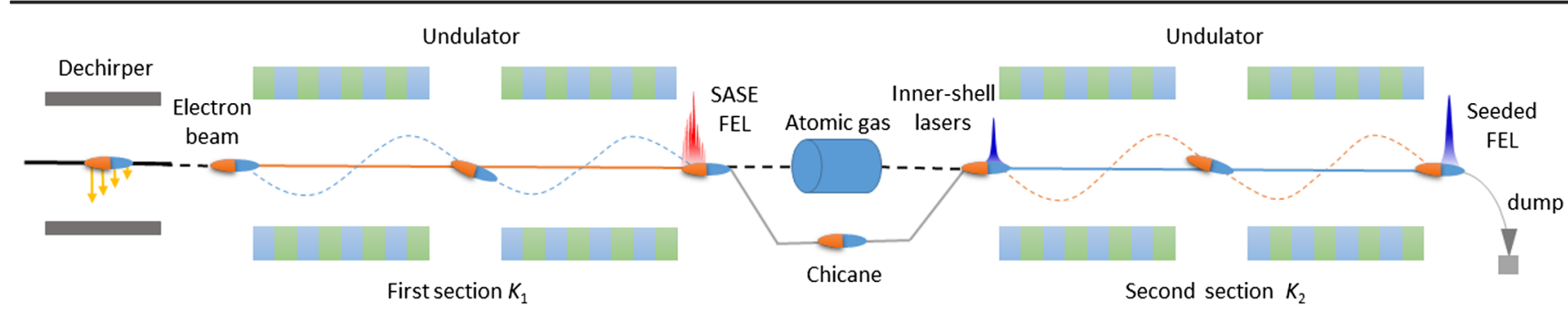

FIG. 1. The seeding scheme: The electron bunch travels off-axis in the dechirper experiencing a strong transverse head-tail kick, electron bunch slices and trajectories are represented in blue for the head and orange for the tail. While passing through the first part of the undulator, the electron bunch orbit is steered to have the bunch tail travelling straightly. A saturated SASE XFEL pulse at energy $E_{1}$ is generated on the bunch tail in the first undulator section tuned to $K_{1}$. After that this XFEL pumps a given neutral atomic gas and it can produce an extremely longitudinal coherent inner-shell XRL at energy $E_{2}$. Then this inner-shell XRL is used as seeding laser to modulate the electron bunch in the head, The bunch orbit is steered, the bunch head is travelling straightly in the second undulator section. The second undulator section tuned to $K_{2}$, its resonance energy is $E_{2}$. A fully saturated light pulse at energy $E_{2}$ is generated on the head.

propagation of atomic inner-shell XRL are discussed in Sec. III. The results of the seeded XFEL is illustrated in Sec. IV, and finally, conclusions are drawn in Sec. V.

\section{THE PRINCIPLES OF SCHEME}

A scheme that exploits the combination of an atomic inner-shell laser and a self-seeding method is presented in Fig. 1. Here the fresh-slice technique was exploited [38], where different temporal slices of an electron bunch laser to saturation in separate undulator sections. The electron bunch travels off-axis in the dechirper experiencing a strong transverse head-tail kick. While passing through the first part of the undulator, the electron bunch tail is traveling straightly. A saturated SASE XFEL pulse at energy $E_{1}$ is generated. After that this XFEL is used to ionize a given neutral atom. It can produce an extremely longitudinal coherent inner-shell XRL at energy $E_{2}$ $\left(E_{1}>E_{2}\right)$. Then this inner-shell XRL is used as seed laser to modulate the electron bunch in the head, at the same

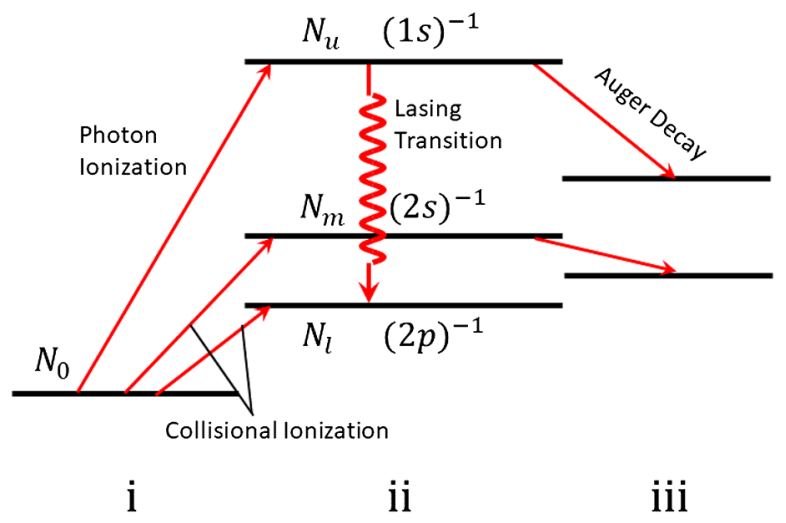

FIG. 2. Schematic diagram of the main inner-shell vacancy levels and atomic processes included in the pumping of innershell XRL. The ground state of the neutral atom and the lasingrelated lower and upper states are represented by $N_{0}, N_{l}, N_{u}$, respectively. time, the bunch orbit is steered again and thus the bunch head travels straightly in the second undulator section. A fully saturated photon beam at energy $E_{2}$ is generated on the head in the second undulator section.

About the generation of atomic inner-shell XRL, it is based on atomic population inversion and driven by rapid $K$ shell photoionization using pump source. This scheme was proposed as early as 50 years ago [27], but has not been experimentally implemented due to the lack of a suitable $\mathrm{x}$-ray pump source. The introduction of XFEL makes it possible to pump new atomic inner-shell XRL. XFEL is used to ionize the core electrons of neutral atoms to produce atomic population inversion, in which excited atoms are attenuated by Auger or radiation. Inner-shell photoionization happens on an ultrafast timescale of a few femtoseconds, the ion temperature in the plasma column is expected to remain close to room temperature during the time of amplification.

Figure 2 shows the considered scheme with the main energy levels related to the inner-shell vacancies and the atomic processes of the direct inner-shell photoionization, Auger decay and the secondary electron collisional ionization included in the photoionization pumped atomic inner-shell XRL. The inner-shell vacancies $(1 s)^{-1}$ are preferentially produced by the x-ray photons with an energy just above the inner-shell ionization threshold. Such light source is now available on the XFEL. The x-ray photons maximize the photoionization cross section of the inner-shell electrons and minimize the secondary electrons production through the photoionization of outer-shell electrons. A population inversion results from the initial photoionization.

\section{ATOMIC INNER-SHELL LASER}

Nowadays, more and more XFEL facilities are under construction and operation all over the world, which can generate extremely ultrashort pulse and high intensity radiation. And currently, the first hard XFEL in China is under construction in Shanghai, namely Shanghai high repetition rate XFEL and extreme light facility (SHINE) [39]. Figure 3 


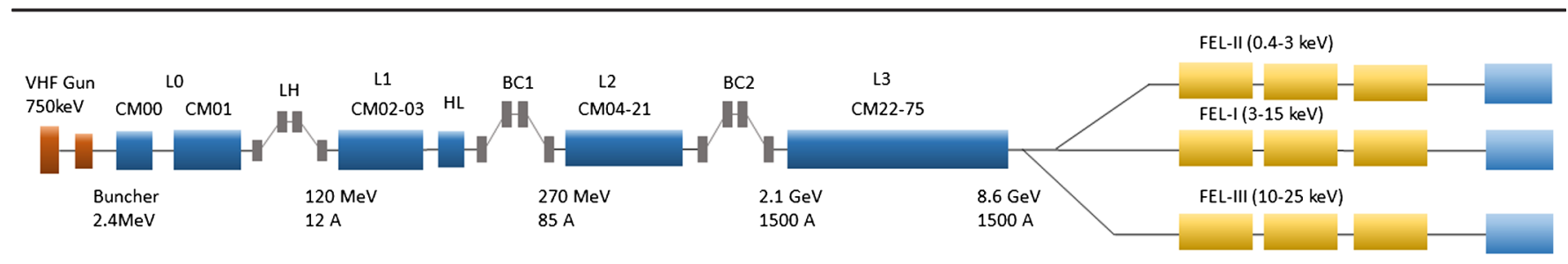

FIG. 3. Machine layout of the SHINE.

shows the layout of the SHINE, SHINE plans to utilize the superconducting linear accelerator to produce quasi-CW relativistic electron beams. The main parameters of the electron beams are as follows: $8 \mathrm{GeV}$ beam energy, $1.5 \mathrm{kA}$ peak current, $0.4 \mathrm{~mm}$-mrad normalized emittance, $0.01 \%$ relative energy spread, and 20-200 $\mathrm{pC}$ bunch charge. As a high repetition rate machine, three FEL lines, i.e., FEL-I, FEL-II, and FEL-III will be equipped simultaneously to cover the full photon range of $0.4-25 \mathrm{keV}$ in the first phase of SHINE.

The studies in this paper are based on the parameters of FEL-II, which is aimed to cover the photon energy of $0.4-3.0 \mathrm{keV}$ by 40 segments main undulator with undulator period of $68 \mathrm{~mm}$ and segment length of $4 \mathrm{~m}$. In order to obtain the fully coherent radiation at soft x-ray region, the baseline design of FEL-II is two-stage seeded FEL scheme. Figure 4 shows the beam phase space at the exit of the SHINE linac. Note that it is essential to use a laser heater to suppress the microbunching instability in order to avoid deteriorating the quality of the beam longitudinal phase space. The electron beam dynamics simulation in the photon-injector is carried out by ASTRA [40] with space charge effects taken into account. ELEGANT [41] is then used for simulation in the reminder of linac. If the RMS shot-toshot variation of the rf amplitude, rf phase, bunch charge, and drive laser arrival time is assumed to be $0.04 \%, 0.04^{\circ}$, $1.5 \%$, and $300 \mathrm{fs}$, from start-to-end simulations, the electron beam jitters at the linac exit are as follows: beam energy jitter

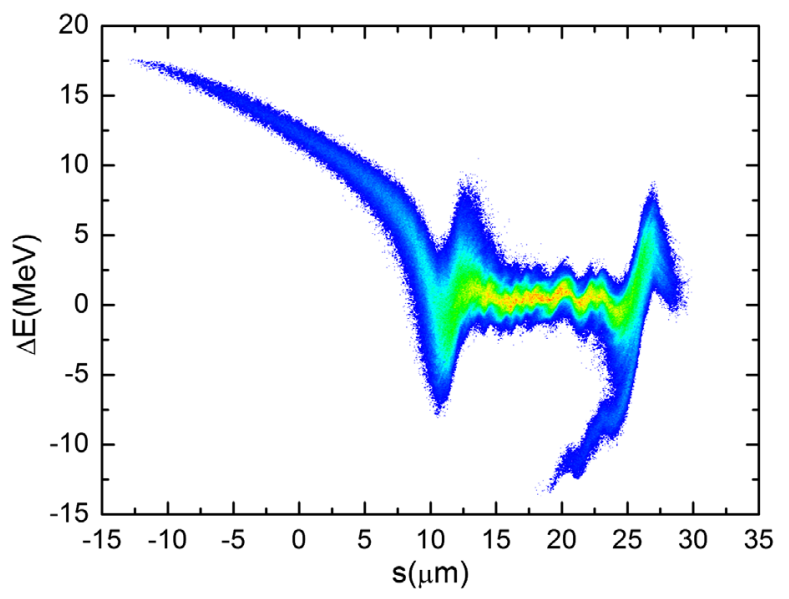

FIG. 4. The beam phase space at the exit of the SHINE linac as expected from ELEGANT simulations, with the laser heater on. of $0.67 \times 10^{-4}$, beam arrival timing jitter of $15.8 \mathrm{fs}$ and a peak current fluctuation of $3.59 \%$. However, from the beam phase space currently available, it is difficult to establish the two-stage seeding mode around $1 \mathrm{keV}$ photon energy. In order to ensure the fully coherence, a better, i.e., more flat and uniform beam phase space should be exploited by reducing the bunch compression factor, and/or an alternative seeding mode should be prepared for backup. Therefore the idea here is that, the upstream 18 segments undulator works in SASE mode, and the downstream 22 segments undulator are seeded by the atomic XRL.

The XFEL simulations are normally carried out by GENESIS [42]. 20 start-to-end time-dependent runs have been conducted and the generated power profile at the end of undulators $(91.26 \mathrm{~m}$ ) are shown Fig. 5. The gray lines display 20 separate runs with different shot noises and result a rms pulse energy jitter about $1.49 \%$, while the red line represents the average value. The typical SASE is expected to be able to generate nearly $77 \mathrm{GW}$ soft X-ray with 8.6 fs (FWHM) pulse duration. The radiation energy along the undulator length is shown on the left side of Fig. 7. As expected for SHINE, we simulated $4.8 \times 10^{12}$ photons at $1 \mathrm{keV}$ photon energy. Such high power pulses can be used directly as a pump source.

This SASE XFEL is used as a seed laser to pump a given atom, and photoionization of the XFEL pulse causes the atom to form holes $[34,35,43,44]$. In this work, we studied

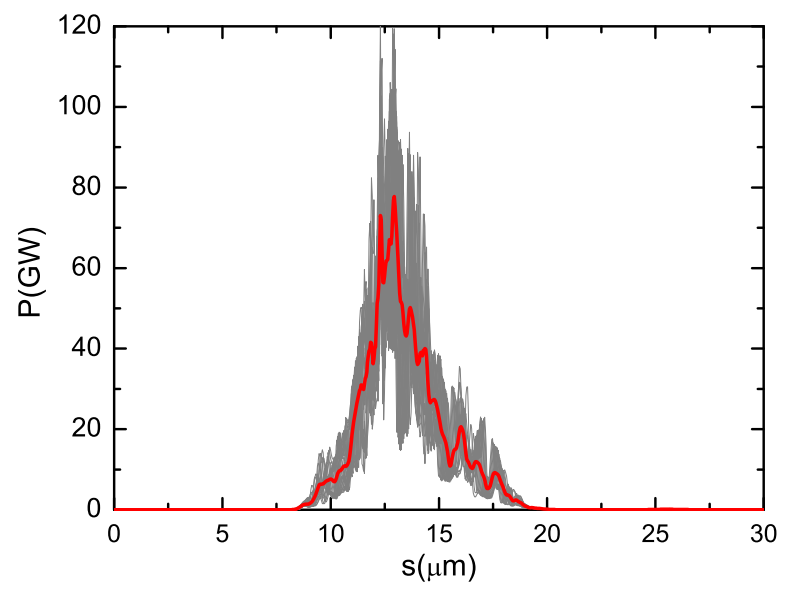

FIG. 5. Temporal power profile at the end of undulators $(91.26 \mathrm{~m})$ for the SASE XFEL radiation in the beam head. Gray curves refer to 20 runs and red curves are their averages. 
XFEL photoionization pumped neon with inner-shell x-ray transitions of $K_{\alpha} 1 s^{-1}-2 p^{-1}(\lambda=1.46 \mathrm{~nm})$. When an electron has been ejected from the $K$ shell of a neon atom, the residual neon ion is left in an excited state which can decay by virtue of an $L$-shell electron dropping down into the $K$-shell. Because the photoionization of the inner shell occurs on the ultrafast time scale of several femtosecond, the ion temperature in the atom keeps close to room temperature. Therefore, the width of the laser is only related to the life of the upper and lower levels. Since the population inversion only occurs in the channels through which the XFEL passes, lasers are expected in a single transverse mode. The lateral divergence is the same size as the divergence of the XFEL beam. The occupancy of upper and lower energy levels is described by solving a rate equation system that describes valence electrons and core photoionization, Auger and radiative decay.

The output characteristics of the XFEL pumped inner shell XRL are discussed. To simulate the output of laser transitions, we applied a one-dimensional model that combines atomic level dynamics with laser propagation and amplification. The XRL intensity $I(z, t)$ is related to the occupancy of the upper and lower layers. The intensity is described by $[34,35]$

$$
\begin{aligned}
\frac{d I_{\mathrm{XRL}}(z, t)}{d t}= & I_{\mathrm{XRL}}(z, t) c n_{A}\left[\sigma_{\mathrm{stim}} N_{U}(z, t)-\sigma_{a b s} N_{L}(z, t)\right] \\
& +\frac{\Omega(z)}{4 \pi} A_{2 p \rightarrow 1 s} N_{U}(z, t) n_{A} c-c \frac{d I_{\mathrm{XRL}}}{d z}
\end{aligned}
$$

where $\Omega(z)=2 \pi\left[1-(L / 2-z) / \sqrt{r^{2}+(L / 2-z)^{2}}\right]$ are the geometrical acceptance angles, allowing propagation in forward directions, $n_{A}$ is the atomic density, and $L$ is the interaction length and $r$ is the diameter spot. Fourth-order

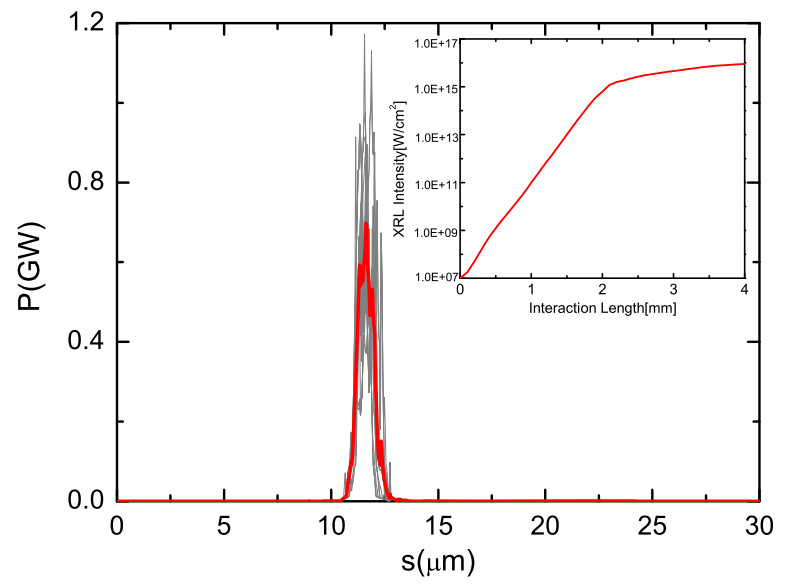

FIG. 6. Temporal power profile of the atomic inner-shell XRL for the $\mathrm{Ne}^{1+} 1 s^{-1}-2 p^{-1}$ line at the exit of the neon gas. Gray curves refer to 20 runs and red curves are their averages. The inset shows output intensity of the XRL for the $\mathrm{Ne}^{1+} 1 s^{-1}-2 p^{-1}$ line as function of the interaction length.
Runge-Kutta algorithm [45] is used to solve these firstorder ordinary differential equations. Due to the longitudinal pumping, lasing occurs only in forward direction. Results for the power profile at the exit are presented in Fig. 6. The inner-shell XRL is able to generate nearly $0.7 \mathrm{GW}$ soft $\mathrm{x}$-ray with $2.6 \mathrm{fs}$ (FWHM) pulse duration. The output pulse energy is $0.95 \mu \mathrm{J}$. Its rms pulse energy jitter is about $1.78 \%$. The energy conversion efficiency is $0.12 \%$. The inset showing the peak intensity of the $\mathrm{Ne}^{1+}$ lasing transition as a function of the interaction length for a neon gas density of $10^{18} \mathrm{~cm}^{-3}$.

\section{SEEDED FEL PERFORMANCE}

In the previous section, we obtained an atomic innershell laser that uses SASE XFEL generated by electron beam tail to pump neutral neon gas atoms. Our selfconsistent gain calculations indicate that average peak power of $0.7 \mathrm{GW}$ radiation can be achieved with femtosecond pulse duration. The electron beam is delayed by $18 \mu \mathrm{m}$ for making the heading electron overlap with seed laser. To extract more energy from electron bunch, tapered undulator technique is used [46]. The magnetic field of 22 segments downstream undulator are finely optimized by genetic algorithm [47] in a steady-state GENESIS simulation. In the optimization, the magnetic field in each undulator segment are set to be equal, i.e., a stepped taper is used.

In order to reveal the real operation of the scheme, 20 start-to-end time-dependent runs have been conducted. The radiation energy along the undulator length $z$ from SASE XFEL to seeded XFEL is shown in Fig. 7(a). The red curve is the average of the gray curves. The energy of the red curve at the end is $1.6 \mathrm{~mJ}$. We can observe an unstable growing of the seeded XFEL power for different cases, this rms pulse energy jitter is about $10.8 \%$. The output soft $\mathrm{x}-$ ray pulse-to-pulse power fluctuation is mainly due to the mismatch between the XFEL power and the setting of the taper.

The generated power profile at the middle and exit of the downstream undulator are shown in Figs. 7(b) and 7(c), respectively. In Fig. 7, we also compared three spectrum distributions between the SASE radiation, and the seeded FEL radiation at the middle and at the end of the undulator line. The gray lines displays 20 separate runs, while the red line represents the average value. The seeded FEL at the end of undulators $(z=111.54 \mathrm{~m})$ is able to generate nearly $210 \mathrm{GW}$ soft X-ray with 7.3 fs (FWHM) pulse duration. The bandwidth is $0.58 \mathrm{eV}$ (FWHM) which corresponds to time-bandwidth product 1.02 . The seeded FEL at the middle of undulators $(z=50 \mathrm{~m})$ is able to generate nearly $65 \mathrm{GW}$ soft X-ray with 5.7 fs (FWHM) pulse duration. The bandwidth is $0.51 \mathrm{eV}$ (FWHM) which corresponds to timebandwidth product 0.7 , at this point, seeded XFEL is almost fully coherent. In the following undulators, the SASE backgrounds from electron beam without seeding 

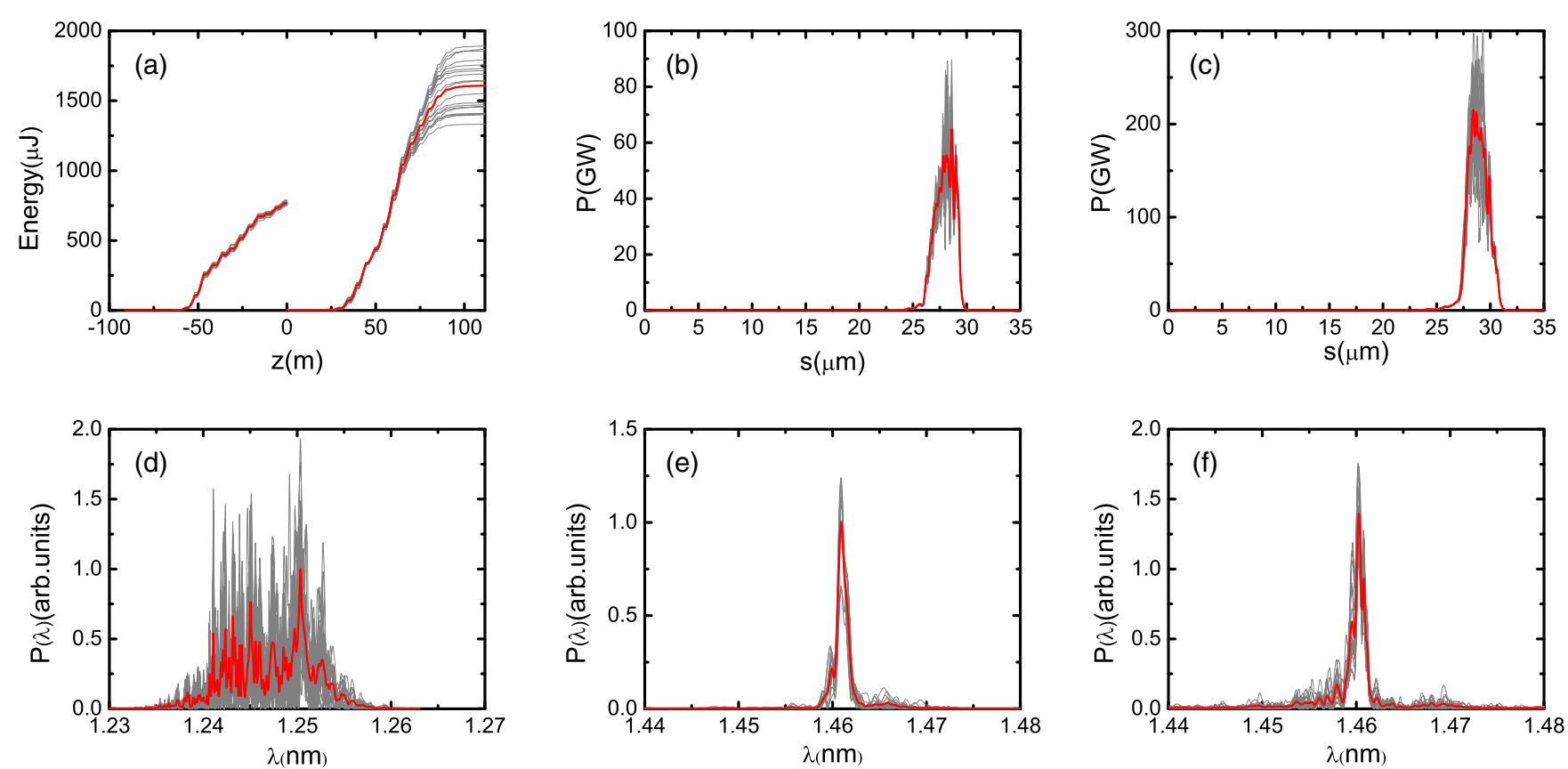

FIG. 7. 20 start-to-end time-dependent runs of the calculation results, the gray lines displays 20 separate runs, while the red line represents the average value. (a) Radiation energy along $\mathrm{z}$ in the SASE XFEL and seeded XFEL stage. (b) Temporal power profile at the middle of undulators $(z=50 \mathrm{~m})$. (c) Temporal power profile at the end of undulators $(z=111.54 \mathrm{~m})$. The peak power of seeded XFEL can reach about $210 \mathrm{GW}$. (d) The spectrum at $z=91.26 \mathrm{~m}$ for the SASE XFEL radiation in the beam head. (e) The spectrum at $z=50 \mathrm{~m}$ for the seeded XFEL. (f) The spectrum at $z=111.54 \mathrm{~m}$ for the seeded XFEL. The bandwidth of the SASE radiation is $7.58 \mathrm{eV}$ and the bandwidth of the seeded XFEL radiation is $0.51 \mathrm{eV}$ in $z=50 \mathrm{~m}$ and $0.58 \mathrm{eV}$ in $111.54 \mathrm{~m}$, respectively.

broaden the bandwidth and the microbunching structures in the electron beam contribute to the spectrum pedestal $[48,49]$. All in all, taking advantage of the overwhelming $0.7 \mathrm{GW}$ fully coherent seeded laser, longitudinal coherence is enhanced at the end of undulators by a factor of 15 with respect to SASE XFEL. Note that the temporal duration of seed laser is nearly $2.6 \mathrm{fs}$, which is shorter than electron beam.

\section{CONCLUSION}

In this paper, a scheme that combines self-seeding method and atomic inner-shell XRL to improve the longitudinal coherence of XFEL is proposed. First, a high peak power SASE is generated by the electron beam tail in the upstream undulators. Then this radiation is used to pump a given neutral neon atom, and thus produce an extremely longitudinal coherent inner-shell XRL. Finally, the electron beam is delayed properly to overlap with seed laser with its heading electrons. It is worth stressing that, while the fresh-slice technique is used for principle illustration in this paper, the proposal can be easily established with in the typical whole bunch self-seeding and/or two bunch self-seeding schemes [50].

Tapered downstream undulators are employed to enhance the output power of seeded soft x-ray. The start-to-end simulations were conducted with parameters from SHINE, which indicates that our scheme is feasible to generate $210 \mathrm{GW}$ nearly fully coherent soft x-ray pulse with 7.3 fs (FWHM) pulse duration. The bandwidth is $0.58 \mathrm{eV}$ (FWHM) which corresponds to time-bandwidth product 1 . Its rms pulse energy jitter is about $10.8 \%$ at the end of the undulator. The seeding scheme is expected to deliver fully coherent radiation to beamline users and enable various experiments. Note that the scheme can be generalized to heavier atoms and therefore even shorter wavelengths in the future. In addition, the new scheme does not use crystals and gratings, which may avoid the thermal loading effects of self-seeding schemes in high repetition rate FEL facilities. Thus, the photon energy absorption and thermal lasing issue in this scheme will be seriously considered in future. An XRL generation and measurement experiment can be accomplished at the experimental station of Shanghai soft x-ray FEL user facility [51], with a focused $\mathrm{x}$-ray beam size of $2 \mu \mathrm{m} \times 3 \mu \mathrm{m}$.

\section{ACKNOWLEDGMENTS}

The authors would like to thank M. Zhang for providing LINAC beam dynamic results of SHINE. This work was partially supported by the National Natural Science Foundation of China (11775293), the National Key Research and Development Program of China (2016YFA0401900), the Young Elite Scientist Sponsorship Program by CAST (2015QNRC001) and Ten Thousand Talent Program. 
[1] D. L. Matthews et al., Demonstration of a Soft X-Ray Amplifier, Phys. Rev. Lett. 54, 110 (1985).

[2] S. Suckewer, C. H. Skinner, H. Milchberg, C. Keane, and D. Voorhees, Amplification of Stimulated Soft X-Ray Emission in a Confined Plasma Column, Phys. Rev. Lett. 55, 1753 (1985).

[3] A. Kondratenko and E. Saldin, Generation of coherent radiation by a relativistic electron beam in an ondulator, Part. Accel. 10, 207 (1980).

[4] R. Bonifacio, C. Pellegrini, and L. M. Narducci, Collective instabilities and high-gain regime in a free electron laser, Opt. Commun. 50, 373 (1984).

[5] P. Emma et al., First lasing and operation of an ångstromwavelength free-electron laser, Nat. Photonics 4, 641 (2010).

[6] T. Ishikawa et al., A compact X-ray free-electron laser emitting in the sub-ångström region, Nat. Photonics 6, 540 (2012).

[7] I. A. Vartanyants et al., Coherence Properties of Individual Femtosecond Pulses of an X-Ray Free-Electron Laser, Phys. Rev. Lett. 107, 144801 (2011).

[8] L. Young et al., Femtosecond electronic response of atoms to ultra-intense X-rays, Nature (London) 466, 56 (2010).

[9] T. Glover et al., X-ray and optical wave mixing, Nature (London) 488, 603 (2012).

[10] T. Togashi et al., Extreme ultraviolet free electron laser seeded by high-order harmonic, Radiat. Phys. Chem. 93, 25 (2013).

[11] G. Geloni, V. Kocharyan, and E. Saldin, A novel selfseeding scheme for hard X-ray FELs, J. Mod. Opt. 58, 1391 (2011).

[12] J. Amann et al., Demonstration of self-seeding in a hardX-ray free-electron laser, Nat. Photonics 6, 693 (2012).

[13] D. Ratner et al., Experimental Demonstration of a Soft X-Ray Self-Seeded Free-Electron Laser, Phys. Rev. Lett. 114, 054801 (2015).

[14] J. Lee, H. S. Chi, M. Yoon, I. Hwang, W. P. Yong, and J. Wu, Optimization of the hard X-ray self-seeding layout of the PAL-XFEL, Nucl. Instrum. Methods Phys. Res., Sect. A 798, 162 (2015).

[15] L. H. Yu, Generation of intense uv radiation by subharmonically seeded single-pass free-electron lasers, Phys. Rev. A 44, 5178 (1991).

[16] L. H. Yu et al., High-Gain Harmonic-Generation FreeElectron Laser, Science 289, 932 (2000).

[17] L. H. Yu et al., First Ultraviolet High-Gain HarmonicGeneration Free-Electron Laser, Phys. Rev. Lett. 91, 074801 (2003).

[18] K. C. Prince, E. Allaria, C. Callegari, R. Cucini, G. D. Ninno, S. D. Mitri, B. Diviacco, E. Ferrari, P. Finetti, and D. Gauthier, Coherent control with a short-wavelength free-electron laser, Nat. Photonics 10, 176 (2016).

[19] G. D. Ninno, D. Gauthier, B. Mahieu, P. R. Ribi, E. Allaria, P. Cinquegrana, M. B. Danailov, A. Demidovich, E. Ferrari, and L. Giannessi, Single-shot spectro-temporal characterization of XUV pulses from a seeded free-electron laser, Nat. Commun. 6, 8075 (2015).

[20] E. Allaria, R. Appio, L. Badano, W. A. Barletta, S. Bassanese, S. G. Biedron, A. Borga, E. Busetto, D. Castronovo, and P. Cinquegrana, Highly coherent and stable pulses from the FERMI seeded free-electron laser in the extreme ultraviolet, Nat. Photonics 6, 699 (2012).

[21] G. Stupakov, Using the Beam-Echo Effect for Generation of Short-Wavelength Radiation, Phys. Rev. Lett. 102, 074801 (2009).

[22] H. Deng and C. Feng, Using Off-Resonance Laser Modulation for Beam-Energy-Spread Cooling in Generation of Short-Wavelength Radiation, Phys. Rev. Lett. 111, 084801 (2013).

[23] C. Feng, H. Deng, D. Wang, and Z. Zhao, Phase-merging enhanced harmonic generation free-electron laser, New J. Phys. 16, 043021 (2014).

[24] K. J. Kim, Y. Shvydko, and S. Reiche, A Proposal for an X-Ray Free-Electron Laser Oscillator with an EnergyRecovery Linac, Phys. Rev. Lett. 100, 244802 (2008).

[25] J. Dai, H. Deng, and Z. Dai, Proposal for an X-Ray Free Electron Laser Oscillator with Intermediate Energy Electron Beam, Phys. Rev. Lett. 108, 034802 (2012).

[26] K. Li and H. Deng, Gain cascading scheme of a freeelectron-laser oscillator, Phys. Rev. Accel. Beams 20, 110703 (2017).

[27] M. Duguay and P. Rentzepis, Some approaches to vacuum UV and X-Ray lasers, Appl. Phys. Lett. 10, 350 (1967).

[28] H. C. Kapteyn, Photoionization-pumped x-ray lasers using ultrashort-pulse excitation, Appl. Opt. 31, 4931 (1992).

[29] T. Axelrod, Inner-shell photoionization-pumped x-ray lasers. Sulfur, Phys. Rev. A 13, 376 (1976).

[30] D. Eder et al., Tabletop x-ray lasers, Phys. Plasmas 1, 1744 (1994).

[31] G. L. Strobel, D. C. Eder, R. A. London, M. D. Rosen, R. W. Falcone, and S. P. Gordon, Innershell photoionized $\mathrm{X}$-ray laser schemes, Proc. SPIE Int. Soc. Opt. Eng. 1860, 157 (1993).

[32] K. Lan, E. Fill, and J. Meyer-ter Vehn, Photopumping of XUV lasers by XFEL radiation, Laser Part. Beams 22, 261 (2004).

[33] J. Zhao, Q. Dong, S. Wang, L. Zhang, and J. Zhang, X-ray lasers from Inner-shell transitions pumped by the Freeelectron laser, Opt. Express 16, 3546 (2008).

[34] N. Rohringer and R. London, Atomic inner-shell x-ray laser pumped by an X-ray free-electron laser, Phys. Rev. A 80, 013809 (2009).

[35] G. Darvasi, C. H. Keitel, and C. Buth, Optical control of an atomic inner-shell x-ray laser, Phys. Rev. A 89, 013823 (2014).

[36] N. Rohringer et al., Atomic inner-shell X-ray laser at 1.46 nanometres pumped by an X-ray free-electron laser, Nature (London) 481, 488 (2012).

[37] H. Yoneda et al., Atomic inner-shell laser at 1.5-ångström wavelength pumped by an X-ray free-electron laser, Nature (London) 524, 446 (2015).

[38] A. A. Lutman et al., Fresh-slice multicolour X-ray freeelectron lasers, Nat. Photonics 10, 745 (2016).

[39] Z.Zhu, Z.Zhao, D. Wang, Z. Liu, R. Li, L. Yin, and Z. Yang, Proceedings of FEL2017, Santa Fe, NM, USA (2017).

[40] K. Flottmann, S. M. Lidia, and P. Piot, Recent improvements to the ASTRA particle tracking code, Office of Scientific and Technical Information Technical Reports 5, 3500 (2003). 
[41] M. Borland, Advanced Photon Source (2000).

[42] S. Reiche, GENESIS 1.3: a fully 3D time-dependent FEL simulation code, Nucl. Instrum. Methods Phys. Res., Sect. B 429, 243 (1999).

[43] N. Rohringer and R. Santra, X-ray nonlinear optical processes using a self-amplified spontaneous emission free-electron laser, Phys. Rev. A 76, 033416 (2007).

[44] R. C. Elton, X-ray Lasers (Elsevier, London, 2012).

[45] P. W. Milonni and J. H. Eberly, Laser Physics (John Wiley and Sons, New York, 2010).

[46] N. Kroll, P. Morton, and M. Rosenbluth, Free-electron lasers with variable parameter wigglers, IEEE J. Quantum Electron. 17, 1436 (1981).

[47] K. Deb, A. Pratap, S. Agarwal, and T. Meyarivan, A fast and elitist multiobjective genetic algorithm: NSGA-II, IEEE Trans. Evol. Comput. 6, 182 (2002).
[48] Z. Zhang, R. Lindberg, W. M. Fawley, Z. Huang, J. Krzywinski, A. Lutman, G. Marcus, and A. Marinelli, Microbunching-instability-induced sidebands in a seeded free-electron laser, Phys. Rev. Accel. Beams 19, 050701 (2016).

[49] K. Zhang, L. Zeng, Z. Qi, C. Feng, and D. Wang, Eliminating the microbunching-instability-induced sideband in a soft x-ray self-seeding free-electron laser, Nucl. Instrum. Methods Phys. Res., Sect. A 882, 22 (2018).

[50] Y. Ding, Z. Huang, and R. D. Ruth, Two-bunch selfseeding for narrow-bandwidth hard $\mathrm{X}$-ray free-electron lasers, Phys. Rev. ST Accel. Beams 13, 060703 (2010).

[51] Z. Zhao et al., in Proceedings of the 2nd International Particle Accelerator Conference, San Sebastiáán, Spain (EPS-AG, Spain, 2011), p. 3011. 